\title{
Totally endoscopic robot-assisted transmyocardial revascularization
}

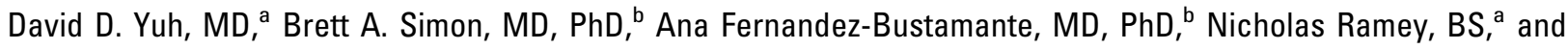
William A. Baumgartner, MD

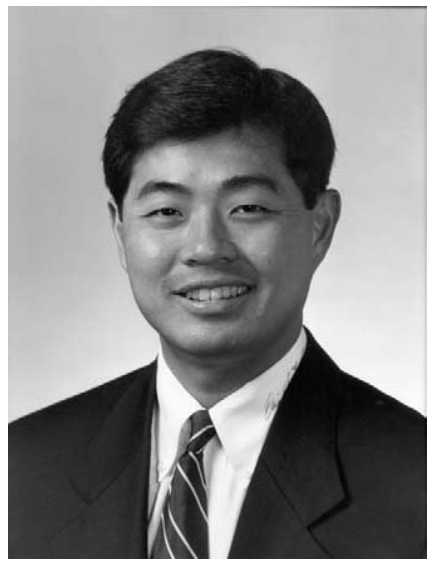

Dr Yuh
From the Division of Cardiac Surgery and the Department of Anesthesia and Critical Care Medicine, ${ }^{\text {b }}$ Johns Hopkins Hospital, Baltimore, Md.

Supported by a grant from CardioGenesis Corporation.

Received for publication Sept 27, 2004; revisions received Oct 27, 2004; accepted for publication Nov 23, 2004

Address for reprints: David D. Yuh, MD, Division of Cardiac Surgery, Johns Hopkins Hospital, 600 North Wolfe Street, Blalock 618, Baltimore, MD 21287-4618 (Email: dyuh@csurg.jhmi.jhu.edu).

J Thorac Cardiovasc Surg 2005;130:120-4

$0022-5223 / \$ 30.00$

Copyright $\odot 2005$ by The American Association for Thoracic Surgery

doi:10.1016/j.jtcvs.2004.11.027
Objective: Laser transmyocardial revascularization is an emerging therapy for intractable angina stemming from diffuse, small-vessel coronary disease not amenable to percutaneous coronary intervention or coronary bypass grafting. Presently, this therapy is delivered through a median sternotomy or left thoracotomy. In this pilot study, we sought to combine the advantages of a dexterous robotic surgical platform with a flexible fiberoptic laser to develop a minimally invasive approach toward transmyocardial revascularization.

Methods: A flexible fiberoptic holmium:yttrium-aluminum-garnet laser probe (CardioGenesis Corporation, Foothill Ranch, Calif), deployed with the da Vinci surgical robotic system (Intuitive Surgical, Sunnyvale, Calif), was used to create transmyocardial channels through all left ventricular wall regions in 5 canine subjects. The channels were localized, quantified, and histologically analyzed to assess distribution, dimensions, and transmurality.

Results: Transmyocardial channels were successfully created in all 6 defined left ventricular wall segments by using this minimally invasive approach without port repositioning, instrument exchange, or probe modifications. Gross pathologic and histologic analyses confirmed the uniform distribution of $1.0-\mathrm{mm}$ transmural channels in all left ventricular regions. No direct pressure, topical hemostatic agents, or suture repairs were required for hemostasis. No significant hemodynamic instability or sustained arrhythmias were encountered at any time during the procedures.

Conclusions: We report the first use of a prototype flexible fiberoptic laser probe deployed by the da Vinci surgical robotic system to successfully perform totally endoscopic off-pump transmyocardial revascularization in a canine model, demonstrating the feasibility, precision, and safety of this approach. Refinement of this minimally invasive technique may reduce the morbidity of open-chest transmyocardial revascularization and facilitate its use as sole therapy or as an adjunct to percutaneous coronary interventions.

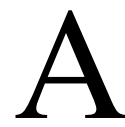
lthough coronary artery bypass grafting (CABG) and percutaneous coronary interventions (PCIs) are proven therapies for relief of angina stemming from ischemic heart disease, there exists a subset of "no-option" patients with intractable angina caused by diffuse, small-vessel coronary disease not amenable to these modalities. Transmyocardial laser revascularization (TMR) has shown some clinical promise in substantially reducing anginal symptoms in these patients either as sole therapy or in conjunction with CABG. ${ }^{1-5}$ Most recently, Allen and colleagues ${ }^{6}$ also demonstrated a significantly reduced risk of late death for no-option patients treated with sole-therapy TMR compared with maximal medical therapy. TMR is currently delivered mainly through a median sternotomy or left thoracotomy. In this study, we sought to combine the unique dexterity afforded by the da Vinci surgical robotic platform with a prototype flexible fiberoptic holmium:yttrium- 

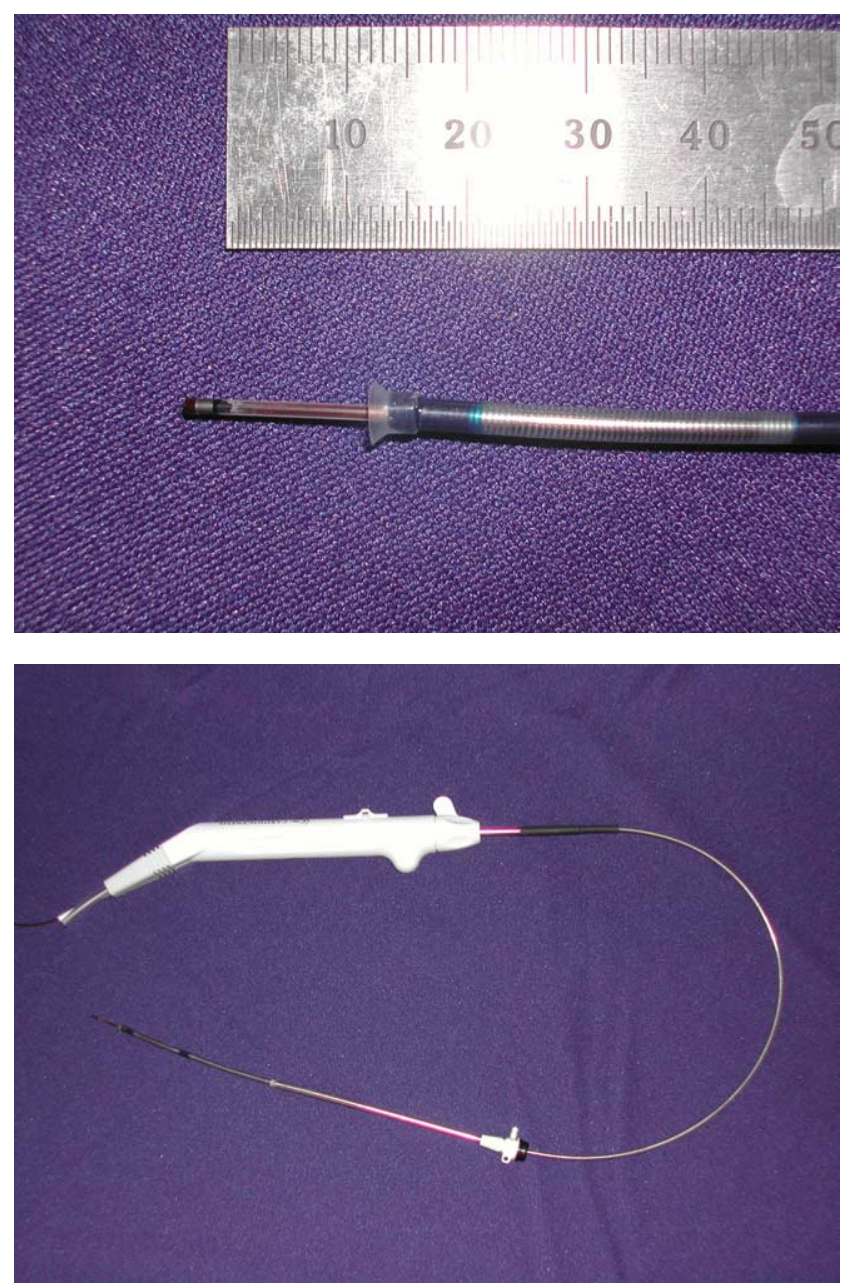

Figure 1. Prototype 1.0-mm CardioGenesis CrystalFlex fiberoptic probe (top) fitted to the commercially available SoloGrip III TMR holmium:yttrium-aluminum-garnet (Ho:YAG) laser handpiece (bottom) (TMR2000; CardioGenesis Corporation, Foothill Ranch, Calif).

aluminum-garnet (Ho:YAG) laser TMR device (CardioGenesis Corporation, Foothill Ranch, Calif) to perform totally endoscopic off-pump TMR in a canine model. We hypothesized that the dexterous characteristics of these 2 systems would facilitate the minimally invasive creation of TMR channels in all segments of the left ventricle.

\section{Materials and Methods}

Adult mongrel dogs of either sex $(n=5)$, weighing between 35 and $40 \mathrm{~kg}$, were used in this experiment under a protocol approved by the Johns Hopkins University Animal Care and Use Committee (Protocol No. DO04M125) on May 11, 2004. All animals received humane care in compliance with the "Principles of Laboratory Animal Care" formulated by the National Society for Medical Research and the "Guide for the Care and Use of Laboratory Animals" prepared by the National Academy of Sciences and
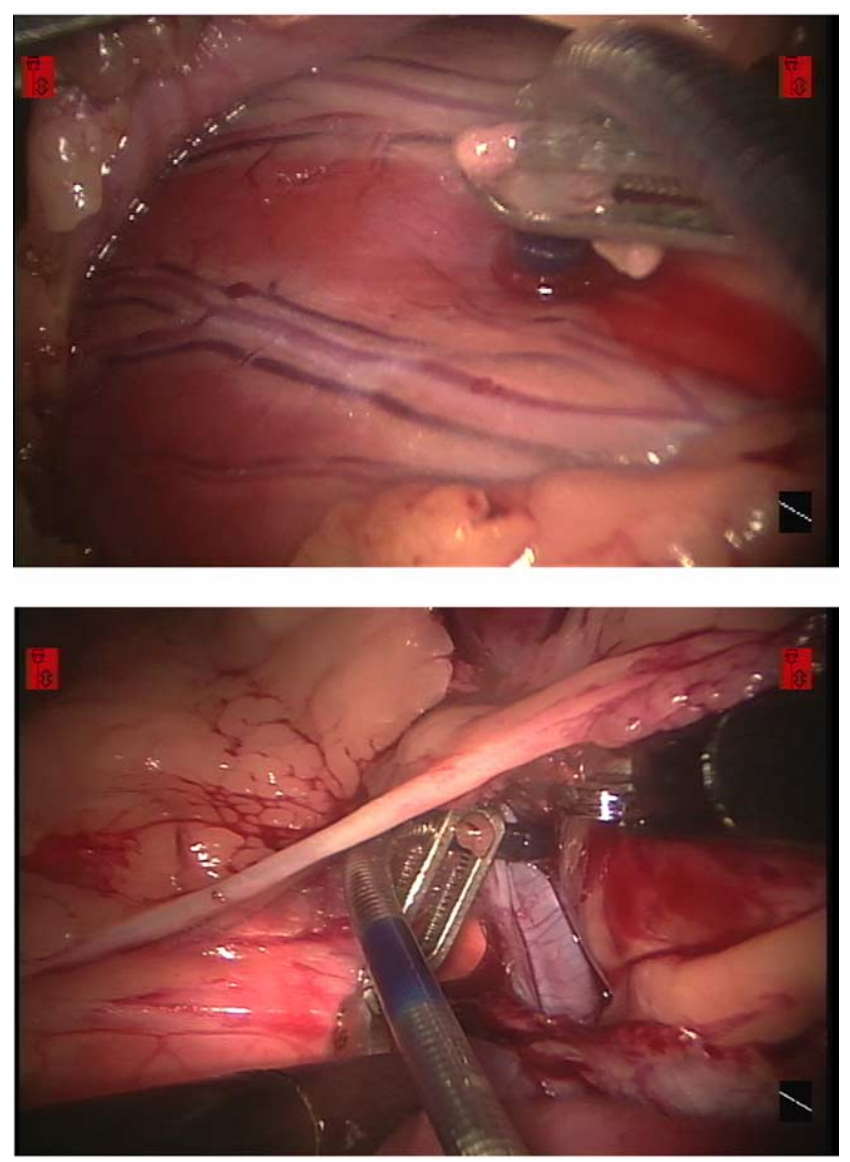

Figure 2. Surgeon's views through the da Vinci system (Intuitive Surgical, Sunnyvale, Calif) master console of robot-assisted, endoscopic transmyocardial revascularization. Transmural channels were created through the anterior (top), anterolateral, apical, posterolateral, posterobasal, and inferior (bottom) walls of the beating canine left ventricle. Note the preserved left phrenic nerve (bottom).

published by the National Institutes of Health (National Institutes of Health Publication No. 85-23, revised 1985). Anesthetic induction was composed of intravenous thiamylal $10 \mathrm{mg} / \mathrm{kg}$ followed by Kottmeier double-lumen endobronchial intubation (Rusch, Duluth, $\mathrm{Ga}$ ) and inhalational anesthesia with isoflurane (1\%-2\%). Each animal was placed in a modified right lateral decubitus position and subjected to selective right-lung ventilation. Systemic arterial pressure was monitored with a femoral arterial line, and venous access was obtained peripherally. Continuous electrocardiographic telemetry and pulse oximetry (V3404 Plus, SurgiVet Inc, Waukesha, Wis) were used.

The da Vinci surgical robotic system (Intuitive Surgical, Sunnyvale, Calif) was used to perform the endoscopic TMR operations. A 0-degree videoscope was introduced into the left thoracic cavity through a 12-mm port (United States Surgical Corp, Norwalk, Conn) placed in the seventh intercostal space along the midscapular line. Two 5-mm da Vinci instrument ports were 


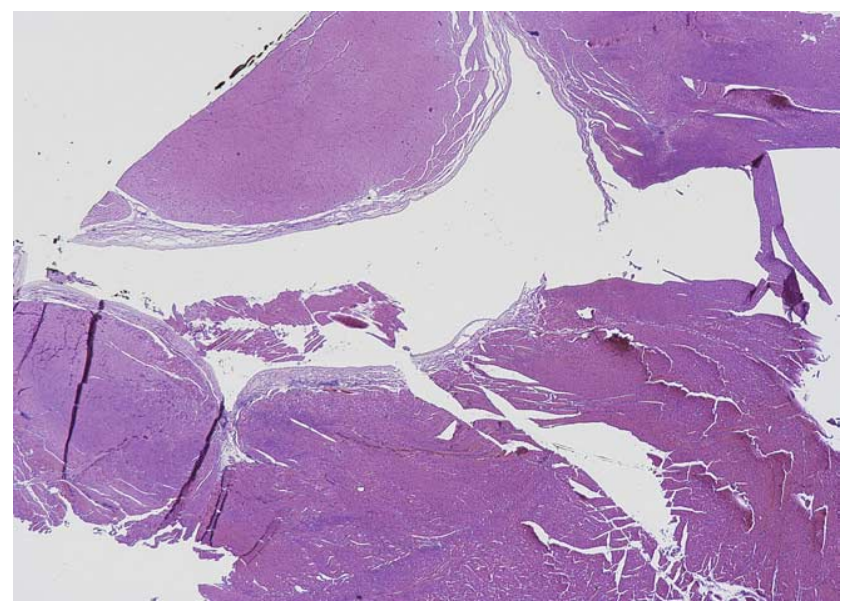

Figure 3. Representative histologic analysis of a transmyocardial channel created with the robot-assisted, minimally invasive da Vinci-CardioGenesis approach. This analysis confirmed the uniform creation of $1.0-\mathrm{mm}$ transmural channels in all sampled regions (hematoxylin-eosin stain, $5 \times$ magnification).

placed in the fifth and ninth intercostal spaces along the posterior scapular line. Finally, a 5-mm "working" port (United States Surgical Corp) was placed in the seventh intercostal space $4 \mathrm{~cm}$ posterior to the videoscopic port. The left thoracic cavity was insufflated with $\mathrm{CO}_{2}$ to pressures ranging from 5 to $10 \mathrm{~mm} \mathrm{Hg}$, and the da Vinci "slave" device was docked to the ports. A da Vinci DeBakey forceps and spatula electrocautery were used to create 2 transverse pericardiotomies, running 1 to $2 \mathrm{~cm}$ anterior and posterior to the left phrenic nerve and extending from base to apex. All wall segments of the left ventricle were easily exposed by grasping the pericardial edges with a single forceps.

A prototype modification of the SoloGrip III TMR Ho:YAG laser handpiece (TMR2000; CardioGenesis Corporation) fitted with a 1.0-mm CardioGenesis CrystalFlex fiberoptic probe (Figure 1) was introduced into the left thoracic cavity through the working port and grasped by 1 of 2 da Vinci DeBakey forceps instruments under videoscopic guidance. Robot-assisted, endoscopic TMR was used to create transmural channels through the anterior, anterolateral, apical, posterolateral, posterobasal, and inferior walls of the beating left ventricle at a density of approximately 1 channel $/ \mathrm{cm}^{2}$ (Figure 2). The laser energy delivered to the myocardium consisted of 7 watt pulses at a $5 \mathrm{~Hz}$ repetition rate with a pulse width of 200 $\mu \sec ( \pm 25 \%)$. The flexible probe was easily maneuverable by the da Vinci instrumentation, and the cupped tip was gently placed on each targeted area, oriented perpendicularly to the epicardial surface without deforming or compressing the left ventricle. Once the probe tip was seated on the epicardial surface, each channel was created by the tableside surgeon by advancing the CrystalFlex fiber, with the SoloGrip handpiece, through the myocardium while discharging the laser pulses through it. Detection of transmural penetration was achieved with auditory feedback to the tableside surgeon. The number of pulses required to create each channel and the number of channels created per ventricular region were recorded. A 30-second pause was taken for ventricular recovery after
TABLE 1. Average number of channels created in each ventricular region by endoscopic robot-assisted transmyocardial revascularization

\begin{tabular}{lc}
\hline $\begin{array}{l}\text { Left ventricular wall region treated } \\
\text { with endoscopic robot-assisted TMR }\end{array}$ & $\begin{array}{c}\text { Mean number of TMR } \\
\text { channels created } \mathbf{\pm} \mathbf{S D}^{*}\end{array}$ \\
\hline Anterior & $9.0 \pm 1.8$ \\
Anterolateral & $7.3 \pm 1.2$ \\
Apical & $5.2 \pm 0.7$ \\
Posterolateral & $6.4 \pm 0.9$ \\
Posterobasal & $8.0 \pm 2.1$ \\
Inferior & $6.7 \pm 1.1$
\end{tabular}

$T M R$, Transmyocardial revascularization. *Five canine subjects.

each group of 10 channels was created. No direct pressure, topical hemostatic agents, or suture repairs were used for any channels. The average operative time, from the initial port placement to the completion of all TMR channels, was 38 minutes.

After the completion of each procedure, each animal was observed for 30 minutes for hemodynamic stability and channel hemostasis. After this period, each animal was euthanized by hyperkalemic cardioplegic arrest under deep inhalational isoflurane anesthesia. A left lateral thoracotomy was performed; channel placement was quantified, localized, and documented; and the hearts were explanted and fixed in $10 \%$ formalin. All experiments were videotaped, and gross pathologic inspection was performed. Targeted myocardial regions were excised and underwent hematoxylin-eosin staining to assess channel distribution, dimensions, and transmurality.

\section{Results}

Each of the 5 animal subjects survived robot-assisted endoscopic TMR. No significant hemodynamic instability or sustained arrhythmias were encountered in any animal subject. As observed with conventional TMR, channel hemostasis occurred spontaneously within several minutes after the creation of the channels, without the need for direct pressure, topical hemostatic agents, or suture closure. No antiarrhythmics, inotropes, blood products, or intravenous fluid boluses were required. Under excellent 3-dimensional videoscopic guidance afforded by the da Vinci system, no epicardial vessels were lacerated with the TMR device. For each channel created, an angle of attack perpendicular to the epicardial plane was readily achievable in all major regions of the left ventricle without port repositioning, instrument exchange, or probe modifications. Approximately 20 to 30 pulses were required to traverse the myocardium. Gross pathologic and histologic analyses confirmed the uniform creation of $1.0-\mathrm{mm}$ transmural channels in all sampled regions (Figure 3 ). The mean number of channels created in each ventricular region is shown in Table 1.

\section{Discussion}

In 1933 Wearn and associates ${ }^{7}$ first described the arterioluminal vessels and intramyocardial sinusoids, which com- 
municate freely with the ventricular cavity. That led to the development of TMR as a means of revascularizing ischemic myocardium by channeling oxygenated blood within the left ventricular cavity into sinusoidal networks serving ischemic regions. Although this mechanism has largely been disproven, ${ }^{8-12}$ significant angina relief observed in nonrandomized TMR trials led to several prospective, randomized 1-year follow-up studies demonstrating angina relief, improved exercise tolerance, decreased rehospitalizations, and improved event-free survival for no-option patients with disabling severe angina refractory to medical management and not amenable to CABG or PCI. ${ }^{1-5} \mathrm{Re}-$ cently, in a 5-year prospective, randomized, multicenter trial, Allen and colleagues ${ }^{6}$ demonstrated sustained longterm angina relief and increased survival in no-option patients receiving primary TMR versus medical therapy.

Currently, most patients receive TMR in conjunction with $\mathrm{CABG}$ or after failed CABG through a median sternotomy or left thoracotomy, targeting myocardial regions not amenable to bypass grafting or PCI. ${ }^{13}$ Far fewer nooption patients undergo sole TMR as an initial surgical procedure, presumably because of a lingering, yet healthy skepticism as to the efficacy of TMR given the following: (1) the lack of a definitive mechanism of action, (2) a perceived placebo effect that has not been strictly excluded in past clinical trials, because of the impracticality of a true surgical "sham" group, and (3) no benefit seen in shamcontrolled, randomized studies using percutaneous myocardial laser revascularization. ${ }^{14}$ Furthermore, diminished survivals have been observed with sole-therapy TMR applied to patients in congestive heart failure or with poor ventricular function. ${ }^{15}$ In this context, few cardiologists and cardiac surgeons are willing to impose the morbidity of a formal thoracotomy or median sternotomy (eg, pain, wound healing, disfigurement) in delivering sole-therapy TMR as an initial operation or even as an adjunct to PCI. To reduce the morbidity-to-benefit ratio of sole-therapy TMR, approaches for minimally invasive TMR have been proposed. deGuzman and colleagues ${ }^{16}$ described the conventional thoracoscopic application of $\mathrm{CO}_{2}$ laser TMR in pigs. Izutani and colleagues ${ }^{17}$ used the Zeus robotic surgical system (Computer Motion, Goleta, Calif) to perform excimer laser (Xe:Cl) TMR in pigs. Although successful to varying degrees, these early attempts could not deliver TMR to all left ventricular regions or required port relocation, instrument exchanges, and/or manual handpiece angle adjustments to do so.

We sought to develop a new minimally invasive, totally endoscopic off-pump approach to TMR, facilitated by a dexterous surgical robotic platform, to more easily deliver TMR to all left ventricular wall regions. The da Vinci surgical robotic system provides wrist-like dexterity and superb stereoscopic visualization within the chest cavity.
We have modified the commercially available CardioGenesis SoloGrip III Ho:YAG fiberoptic laser probe, permitting it to be introduced into the chest cavity through a $5-\mathrm{mm}$ thoracoscopic port and easily manipulated across a wide range of angles by the da Vinci instrumentation. We selected the Ho:YAG laser over other commercially available $\mathrm{CO}_{2}$ lasers because with the latter, laser energy is not transmitted fiberoptically, but rather through relatively fixed reflective elements. Combining the da Vinci system's robotic dexterity with the Ho:YAG laser's flexible fiberoptic conduit greatly facilitates positioning the probe at the precise location and perpendicular orientation to all left ventricular regions for optimal TMR channel creation. Although we believe this approach compares favorably with conventional thoracoscopic techniques in this regard, we did not perform the same procedure with standard thoracoscopic instruments to make objective comparisons.

Histologic and gross confirmation of transmural channel penetration and distribution in all left ventricular wall segments were achieved in this study. The excellent visualization and precise maneuverability afforded by this approach also reduces the chance of inadvertent injury to epicardial vessels. In more than 200 channels created in these experiments, no epicardial vessel was lacerated.

Single-lung ventilation with gentle $\mathrm{CO}_{2}$ insufflation greatly facilitates off-pump exposure of the heart for this totally endoscopic approach. In turn, avoiding heparinization associated with cardiopulmonary bypass greatly promotes hemostasis. In these pilot studies, uniform spontaneous channel hemostasis without the aid of direct pressure, topical hemostatic agents, or suture closure was achieved. Furthermore, no sustained arrhythmias or hemodynamic instability was noted in any of the preparations. We recognize that this stability may not always be afforded by an ischemic ventricle; therefore, we anticipate the mandatory use of external defibrillator pads and cardiopulmonary bypass standby for this closed-chest approach.

Finally, we recognize that most animal models do not anatomically replicate the human thorax; however, we did find that, in our canine preparation, the left ventricle lies within several centimeters to the chest wall, as it does in humans with dilated cardiomyopathies. Despite this, we were able to easily direct the TMR probe to all left ventricular regions. This is an advantage afforded by our robotic approach and may prove to obviate the need for additional exposure devices used in other off-pump operations.

In summary, we describe our early experimental experience with a totally endoscopic, robot-assisted off-pump approach for delivering TMR therapy. By combining the advantages of a dexterous robotic surgical system with a prototypic modification of a commercially available flexible fiberoptic Ho:YAG TMR system, we demonstrate the feasibility, precision, and safety of this approach. The targeted 
aims of this strategy are to substantially reduce the morbidity of TMR therapies arising from open-chest approaches and to facilitate the minimally invasive application of TMR as initial sole therapy or as an adjunct to primary PCI, addressing ischemic myocardial regions supplied by nonstentable coronary arteries. Further developmental studies appear to be warranted.

We acknowledge Phillip Randall Brown, DVM, MSc, Sue Eller, RVT, and Chrissy Sheckells of the Johns Hopkins Minimally Invasive Surgical Training Center for their technical assistance. We also thank John McIntyre and Chuck Scarano of CardioGenesis Corporation for the development of the prototype modification of the SoloGrip III Ho:YAG fiberoptic laser probe.

\section{References}

1. Allen KB, Dowling RD, Fudge TL, et al. Comparison of transmyocardial revascularization with medical therapy in patient with refractory angina. N Engl J Med. 1999;341:1029-36.

2. Aaberge L, Nordstrand K, Dragsund M, et al. Transmyocardial revascularization with $\mathrm{CO}_{2}$ laser in patients with refractory angina pectoris. J Am Coll Cardiol. 2000;35:1170-7.

3. Burkhoff D, Schmidt S, Schulman SP, et al. Transmyocardial revascularization compared with continued medical therapy for treatment of refractory angina pectoris. Lancet. 1999;354:885-90.

4. Frazier OH, March RJ, Horvath KA. Transmyocardial revascularization with a carbon dioxide laser in patients with end-stage coronary disease. N Engl J Med. 1999;341:1021-8.

5. Schofield PM, Sharples LD, Caine N, et al. Transmyocardial laser revascularization in patients with refractory angina: a randomized controlled trial. Lancet. 1999;353:519-24.
6. Allen KB, Dowling RD, Angell WW, et al. Transmyocardial revascularization: 5-year follow-up of a prospective, randomized multicenter trial. Ann Thorac Surg. 2004;77:1228-34.

7. Wearn JT, Mettier SR, Klumpp TG, Zschiesche LJ. The nature of the vascular communications between the coronary arteries and the chambers of the heart. Am Heart J. 1933;2:143-64.

8. Sigel JE, Abramovitch CM, Lytle BW, Ratliff NB. Transmyocardial laser revascularization: three sequential autopsy cases. J Thorac Cardiovasc Surg. 1998;115:1381-5.

9. Burkhoff D, Fisher PE, Apfelbaum M, et al. Histologic appearance of transmyocardial laser channels after 4 1/2 weeks. Ann Thorac Surg. 1996;61:1532-5.

10. Kohmoto T, Fisher PE, Gu A, et al. Does blood flow through holmium: YAG transmyocardial laser channels? Ann Thorac Surg. 1996;61: 861-8.

11. Kohmoto T, Fisher PE, Gu A, et al. Physiology, histology, and two week morphology of acute myocardial channels made with a $\mathrm{CO} 2$ laser. Ann Thorac Surg. 1997;63:1275-83.

12. Gassler N, Wintzer HO, Stubbe HM, et al. Transmyocardial laser revascularization: histological features in human nonresponder myocardium. Circulation. 1997;95:371-5.

13. Allen KB, Dowling RD, Schuch DR, et al. Adjunctive transmyocardial revascularization: 5-year follow-up of a prospective, randomized trial. Ann Thorac Surg. 2004;78:458-65.

14. Saririan M, Eisenberg MJ. Myocardial laser revascularization for the treatment of end-stage coronary artery disease. $J$ Am Coll Cardiol. 2003;41:173-83.

15. Guleserian KJ, Maniar HS, Camillo CJ, et al. Quality of life and survival after transmyocardial laser revascularization with the holmium:YAG laser. Ann Thorac Surg. 2003;75:1842-7.

16. deGuzman BJ, Lautz DB, Chen FY, et al. Thoracoscopic transmyocardial laser revascularization. Ann Thorac Surg. 1997;64:171-4.

17. Izutani H, Gill IS, Svanidze O. Robotically assisted endoscopic transmyocardial laser revascularization. Can J Cardiol. 2004;20: 907-9. 